\title{
Pyrene-p-tert-butylcalixarenes inclusion complexes formation: a surface photochemistry study
}

\author{
T. J. F. Branco, ${ }^{a}$ L. F. Vieira Ferreira, ${ }^{* a}$ A. M. Botelho do Rego, ${ }^{a}$ A. S. Oliveira ${ }^{a}$ and J. P. Da Silva ${ }^{a, b}$ \\ Received 21st June 2006, Accepted 27th September 2006 \\ First published as an Advance Article on the web 10th October 2006 \\ DOI: $10.1039 /$ b608833c
}

Diffuse reflectance and luminescence techniques were used to study the photophysics and photochemistry of pyrene within $p$-tert-butylcalix[n]arenes with $n=4,6$, and 8 , and to study their ability to form inclusion complexes in heterogeneous media. Evidences for inclusion complex formation were found for the three hosts under study. Ground state diffuse reflectance results have shown the formation of ground state dimers of pyrene inside the cavity of calix[6]arene and calix[8]arene, with this feature much more evident for calix[6]arene. For calix[4]arene, only a monomer fits inside the cavity and the presence of pyrene microcrystals outside the cavity was detected. A luminescence lifetime distribution analysis was performed, revealing the presence of prompt emissions from the pyrene microcrystals outside the cavity in the case of calix[4]arene and from the constrained dimers inside the cavities of calix[6]arene and calix[8]arene. Transient absorption results have shown the presence of pyrene radical cation and also of trapped electrons for the three hosts under study. The formation of the phenoxyl radical of the calixarene following the laser pulsed excitation of pyrene at $355 \mathrm{~nm}$ is increased for calix[6]arene and calix[8]arene. This feature is particularly relevant for calix[6]arene, suggesting a very favourable situation for the hydrogen atom abstraction to occur. The analysis of the degradation products revealed the presence of hydroxypyrene as a major photodegradation product for the three hosts. Dihydro-hydroxypyrene was also formed in the case of calix[6]arene and calix[8]arene. The formation of the calixarene's phenoxyl radical and subsequent hydrogen abstraction is consistent with the formation of dihydro-dihydroxypyrene.

\section{Introduction}

Calixarenes are macrocyclic phenol-formaldehyde polycondensates that possess calix shaped intramolecular hydrophobic cavities. $^{1-4}$ These $\left[1_{n}\right.$ ] class cyclophanes have become popular substrates, being capable of forming inclusion complexes with both neutral and ionic guest molecules in a selective way. ${ }^{5,6}$ The applications for this family of compounds are therefore increasing in domains such as supramolecular and environmental chemistry. Industrially, calixarenes have been used in catalysis, chromatographic separations and waste recovery. ${ }^{4-8}$

The cyclic structure of calixarenes allows for several conformations that increase in number with the number of phenolic units composing the ring. Thus, calix[4]arene has 4 conformers, calix[6]arene has 8, and calix[8]arene can assume up to 16 different conformations ${ }^{9-11}$ (see Scheme 1). All these conformers are interconvertible in solution although some conformations are more stable than others. The cone conformation in calix[4]arene is usually stabilized by hydrogen bonds between the 4-hydroxyl groups in the lower rim of the phenolic ring. However, in some solvents like acetone or acetonitrile, this cyclic array does not exist and conformational liberty increases. ${ }^{7}$

${ }^{a}$ Centro de Química-Física Molecular, Instituto Superior Técnico, 1049-001, Lisboa, Portugal.E-mail: luisfilipevf@ist.utl.pt

${ }^{b}$ F. C. T., Universidade do Algarve, Campus de Gambelas, 8005-139, Faro, Portugal
The ability to form endo inclusion complexes is directly related to the size of the cavity and to its aromatic character. A cavity that in a regular cone conformation is aromatic and hydrophobic becomes less hydrophobic if one or more phenolic units are inverted, placing the hydroxyl groups closer to the upper rim.

The dimensions of the methylenic bridge rings of the cone conformation have been measured to be about 7.2, 9.3 and 10.7 $\AA$ for p-tert-butylcalixarenes [4], [6] and [8] in X-ray structures, respectively. ${ }^{12}$ The dimension of the cavity however, depends strongly not only on the conformation but also on the angle of the phenolic units with the inner axis of the calix. This angle changes to accommodate the different shapes and dimensions of the various probes. ${ }^{8,13}$

Review papers about the ability of calixarenes to form inclusion complexes with organic and inorganic guests have been presented, ${ }^{5,7,14,15}$ as well as studies about clathrate formation. ${ }^{13}$

Their versatility as host molecules results not only from their different sized cavities, but also from their easy functionalization of both upper and lower rims allowing for the introduction of functional groups, sensors, switches, or the fixation to polymers. ${ }^{8,14,16,17}$ Industrial applications for this family of compounds are therefore expected to grow in the next years.

In this work, pyrene has been used as a probe and p-tertbutylcalix[4], [6], and [8]arenes as host molecules. Polycyclic aromatic hydrocarbons (PAHs) are well known persistent pollutants with toxic and carcinogenic activity. ${ }^{18}$ Pyrene is one of the PAHs listed as priority pollutants by the Environmental Protection Agency. ${ }^{18}$ In spite of the fact that water soluble calix $[n]$ arenes 


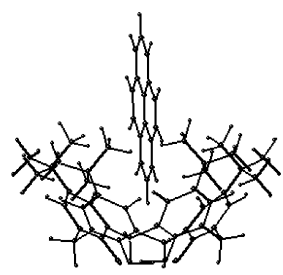

a)

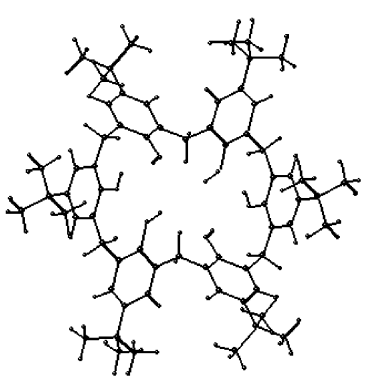

b )

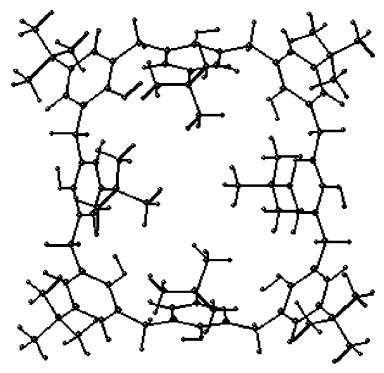

c)

Scheme 1 (a) Pyrene, the guest molecule used in this work, within $p$-tert-butylcalix[4]arene in the cone conformation, (b) $p$-tert-butylcalix[6]arene in the cone conformation and (c) p-tert-butylcalix[8]arene in the 1,2,3,4 alternate cone (or pinched cone) conformation.

$(n=4)$ do not form stable inclusion complexes with pyrene, ${ }^{8}$ the study of inclusion complex formation for the parent compounds (with $n=4,6$, and 8 ) for the case of solid powdered samples, remains to be performed.

In this way, we studied the ability of solid powdered calixarenes to entrap these neutral molecules by the use of diffuse reflectance and luminescence techniques. These surface analysis techniques have recently been used by our group for the study of several heterogeneous systems ${ }^{19}$ and by using them we aim to contribute to the development of cleaning and filtering applications of these hosts towards PAHs.

\section{Experimental}

\section{Materials}

Pyrene, $p$-tert-butylcalix[4]arene, $p$-tert-butylcalix[6]arene, and $p$ tert-butylcalix[8]arene, (all from Aldrich) were used without any purification. Chloroform, ethanol (Merck, Uvasol grade), methanol and acetonitrile (Merck, Lichrosolv) were also used as received. Water was deionised and distilled.

\section{Sample preparation}

The solid powdered samples pyrene-calixarene (molar ratios 1 : $1 ; 1: 2.5 ; 1: 5 ; 1: 10 ; 1: 25 ; 1: 50)$ used in this work were prepared using the solvent evaporation method. ${ }^{19}$ This method consists of the addition of a solution containing the probe to a saturated solution of the calixarene $\left(\sim 10^{-2} \mathrm{M}\right)$, both in chloroform. The resulting mixture was magnetically stirred for at least $24 \mathrm{~h}$ and then allowed to evaporate in a fume cupboard. The final solvent removal was performed overnight in an acrylic chamber with an electrically heated shelf (Heto, Model FD 1.0110) with temperature control $\left(30 \pm 1{ }^{\circ} \mathrm{C}\right)$ and under moderate vacuum at a pressure of $c a \cdot 10^{-3}$ Torr. The evaluation of the existence of final traces of solvent was monitored by the use of FTIR spectra. After some time and due to handling, samples tend to accumulate residual amounts of water. Pyrene-cellulose samples were prepared by the same method, using ethanol instead of chloroform. Mechanical mixtures were prepared simply by grinding the probe and the substrate in a mortar with an agate pestle (ten minutes each time and about one hour in the total).

The choice of an adequate solvent is very important because solvent molecules may compete with the probe in the inclusion complex formation process.

\section{Methods}

Ground state diffuse reflectance absorption spectra (GSDR). Ground state absorption spectra for the solid samples were recorded using an OLIS 14 spectrophotometer (based on a Cary 14) with a diffuse reflectance attachment. Further details are given elsewhere. ${ }^{20,21}$ The diffuse reflectance attachment used in the Cary 14 is described in detail in ref. 21c. The integrating sphere includes a baffle and can be used in two different positions, allowing the detection of the specular light or avoiding it. In this way, we obtained $1-2 \%$ of specular radiation only. The cut-on filter (UG5) used in this work was supplied by Corion.

Steady state luminescence (SSL). Corrected fluorescence emission spectra (steady state) were obtained with a home-made apparatus described elsewhere. ${ }^{22}$

Diffuse reflectance laser flash photolysis (DRLFP) system and laser induced luminescence (LIL). Schematic diagrams of the LIL system and DRLFP systems are presented in ref. 23. Laser flash photolysis experiments were carried out with the third harmonic of a YAG laser $(355 \mathrm{~nm}, c a .6 \mathrm{~ns}$ FWHM, $\sim 10-30 \mathrm{~mJ}$ per pulse) from B. M. Industries (Thomson-CSF), model Saga 1210 , in the diffuse reflectance mode. ${ }^{19 a, 23 b}$ The light arising from the irradiation of solid samples by the laser pulse is collected by a collimating beam probe coupled to an optical fibre (fused silica) and is detected by a gated intensified charge coupled device (ICCD, Oriel model Instaspec V). The ICCD is coupled to a fixed imaging compact spectrograph (Oriel, model FICS 77440). The system can be used either by capturing all light emitted by the sample or in a time-resolved mode by using a delay box (Stanford Research Systems, model D6535). The ICCD has high speed (2.2 ns) gating electronics and intensifier and works in the 200-900 nm wavelength range. Time resolved absorption and emission spectra are available in the nanosecond to second time range. ${ }^{19 a, 23 b}$ Transient absorption data are reported as percentage of absorption ( $\% \mathrm{Abs}$.), defined as $100 \Delta J_{t} / J_{\mathrm{o}}=\left(1-J_{t} / J_{\mathrm{o}}\right) 100$, where $J_{\mathrm{o}}$ and $J_{t}$ are diffuse reflected light from sample before exposure to the exciting laser pulse and at time $t$ after excitation, respectively. ${ }^{19 a, 23 b}$

For the laser induced luminescence experiments a $\mathrm{N}_{2}$ laser (PTI model 2000, ca. 600 ps FWHM, $\sim 1.3 \mathrm{~mJ}$ per pulse) was also used.

Irradiation and product analysis. Photodegradation studies under lamp irradiation were performed in a reactor previously used to study the photochemistry of pesticides. ${ }^{23 c, 24}$ The samples were irradiated at $254 \mathrm{~nm}$ using a $400 \mathrm{~W}$ medium-pressure mercury 
lamp (Applied Photophysics) without filters. Refrigeration was performed using an external cooling bath. ${ }^{24 a}$ Laser irradiation at $355 \mathrm{~nm}$ was also used. The degradation products were analysed after extraction with acetonitrile or methanol (a known weight of sample in a known volume of solvent) followed by centrifugation. Photolysis was followed by HPLC using a MerckHitachi 655A-11 chromatograph equipped with detectors 655A22 UV and Shimadzu SPD-M6A Photodiode Array. A column LiChroCART 125 (RP-18, $5 \mu \mathrm{m}$ ) Merck was used and the runs were performed using water-acetonitrile mixtures. The extracts were also analysed by GC-MS using a Hewlett Packard 5890 Series II gas chromatograph with a 5971 series mass selective detector (EI $70 \mathrm{eV}$ ). A Chrompack CP-WAX 58CB capillary column with $25 \mathrm{~m}$ and $0.25 \mathrm{~mm}$ I.D. was used. A DB-1 capillary column with $30 \mathrm{~m}$ and $0.25 \mathrm{~mm}$ I.D. (J \& W Scientific) was also used. The initial temperature $70^{\circ} \mathrm{C}$ was kept during $5 \mathrm{~min}$ and then a raising rate of $5^{\circ} \mathrm{C} \mathrm{min}{ }^{-1}$ was used until $250{ }^{\circ} \mathrm{C}$.

\section{Results and discussion}

\section{Ground state diffuse reflectance (GSDR)}

Fig. 1 shows the ground state absorption spectra of pyrenecalix $[n]$ arene samples prepared in concentrations ranging from 1 : 50 to $1: 1$ guest : host molar ratio. As the concentration increases, different behaviours are observed for the three hosts under study.

For calix[4]arene (Fig. 1(a)), an increase in the $\mathrm{S}_{1} \leftarrow \mathrm{S}_{0}\left({ }^{1} \mathrm{~L}_{\mathrm{b}}\right)$ transition band around $372 \mathrm{~nm}$ is observed. Some authors believe this absorption is due to ground state dimers (also known as bimolecular ground state associations, BGSAs ${ }^{25,26}$ and others argue that it is due to the presence of pyrene microcrystals. ${ }^{27}$

Our results support the conclusion that in the case of calix[4]arene, this absorption is due to the presence of microcrystals for the following reasons: the growth of this absorption band was observed by us in diluted mechanical mixtures of pyrene microcrystals on an inert surface (data not shown). Also, in a previous study using the same samples of pyrene in calix[4]arene, the presence of pyrene microcrystals was confirmed ${ }^{28}$ Finally, the calix[4]arene samples do not exhibit the same changes that are observed for calix[6]arene and calix[8]arene in the $\mathrm{S}_{2} \leftarrow \mathrm{S}_{0}\left({ }^{1} \mathrm{~L}_{\mathrm{a}}\right)$ transition zone.

For these two hosts, as the concentration increases two important phenomena emerge: a notorious broadening at $324 \mathrm{~nm}$, and a bathochromic shift $(2-3 \mathrm{~nm})$ of the band peaking at $337 \mathrm{~nm}$. These same changes were observed for pyrene adsorbed onto other substrates and have been related to the presence of ground state dimers. ${ }^{25,26,29}$ Studies with microcrystalline cellulose revealed that pyrene dimers were formed during the solvent evaporation process. In these works, the guest molecules stay entrapped after solvent removal, ${ }^{20}$ and as the concentration increases, ground state dimers are also entrapped into a specific conformation. Fig. 1(b) and (c) show both the bathochromic shift and the broadening at $324 \mathrm{~nm}$, revealing the formation of these dimers and the creation of new absorbing species for calix[6]arene and calix[8]arene, respectively.

The consistent changes in the absorption spectra (along with other evidence discussed further in this paper) suggest that the calix[6]arene's hydrophobic cavity has the ideal dimensions to entrap the pyrene ground state dimer. The small changes observed in the $\mathrm{S}_{1} \leftarrow \mathrm{S}_{0}$ transition zone also reveal the presence
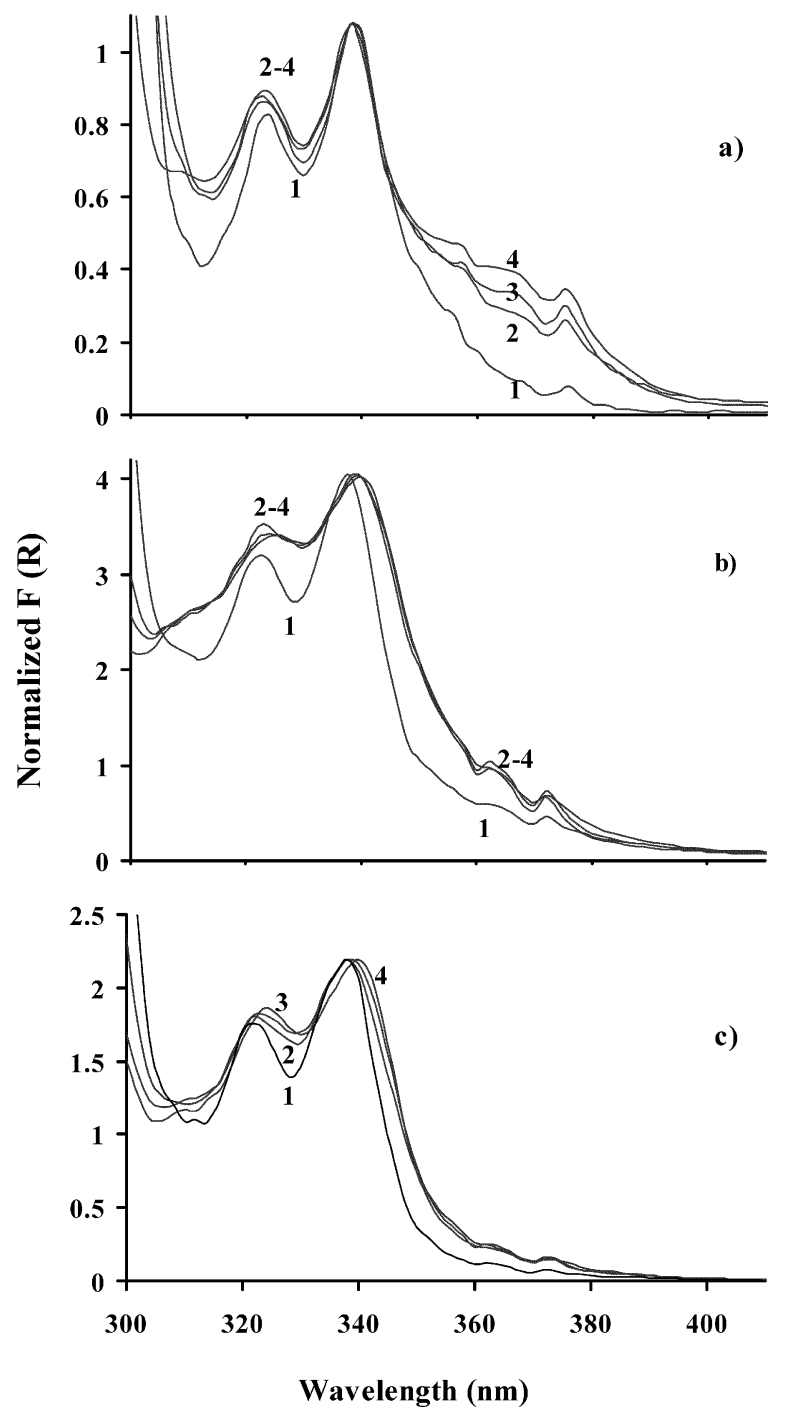

Fig. 1 Normalized remission function of the GSDR of pyrene adsorbed on calix[4]arene, calix[6]arene and calix[8]arene, (a), (b) and (c), respectively, in the molar ratios guest : host of $1: 50$ (1), $1: 10$ (2), $1: 5$ (3) and $1: 1$ (4).

of small amounts of pyrene microcrystals outside the cavities of calix[6]arene and calix[8]arene.

For calix[8]arene, due to the multiplicity of possible conformations, the changes are more gradual with the increase of the pyrene concentration. In the case of calix[4]arene, the cavity has the appropriate size to entrap the pyrene monomer, but not the ground state dimer.

\section{Steady state luminescence}

Pyrene molecules exhibit five vibronic bands in the region from 372 to $392 \mathrm{~nm}$, referred to as $I_{1}$ (or $0-0$ ), $I_{2}, I_{3}, I_{4}$ and $I_{5}$. While the third band $\left(\mathrm{I}_{3}\right)$ is almost constant in both absorption and fluorescence spectra, the intensity of the $I_{1}$ band is enhanced by increasing media polarity. ${ }^{25}$ This symmetry allowed and vibronically forbidden transition is stronger in polar environments due to symmetry lowering perturbations allowing for vibronic coupling between $S_{1}$ and $S_{2}$ excited electronic states. ${ }^{25,30}$ The decrease of the $\mathrm{I}_{3} / \mathrm{I}_{1}$ ratio with media polarity allows us to compare 
the three different environments pyrene experiences in the cavities of the three hosts.

Fig. 2(a), (b) and (c) show the SSL spectra of pyrene in calix[4]arene, calix[6] arene and calix[8]arene, respectively, normalized at the $\mathrm{I}_{3}$ band. The $\mathrm{I}_{3} / \mathrm{I}_{1}$ ratios are $1.3,1.6$ and 1.2 for pyrene in calix[4]arene, calix[6] arene and calix[8]arene, respectively.
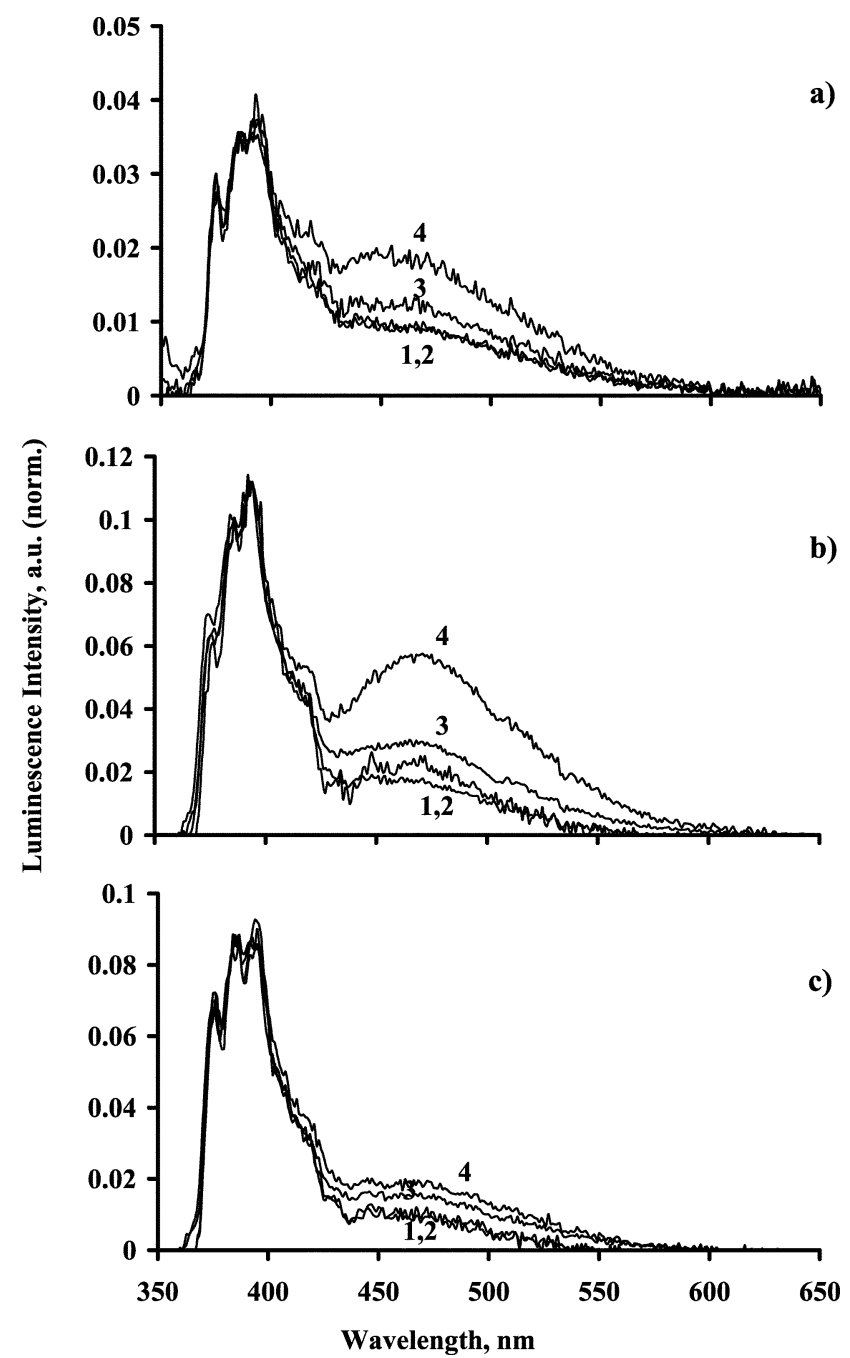

Fig. 2 Steady state fluorescence spectra of pyrene adsorbed onto calix[4]arene, calix[6]arene and calix[8]arene, (a), (b) and (c), respectively, in the molar ratios guest : host of $1: 50$ (1), $1: 10$ (2), $1: 5$ (3) and $1: 1$ (4); $\lambda_{\text {exc. }}=337 \mathrm{~nm}$.

The highest $I_{3} / I_{1}$ ratio indicates that calix[6]arene provides the least polar environment for the pyrene molecules, which is consistent with the presence of a dimer inside the hydrophobic cavity. For calix[8]arene, despite the presence of the dimer, the cavity's polarity is increased by the multiple conformations that place some of the hydroxyl groups close to the cavity. In the case of calix[4]arene, the $\mathrm{I}_{3} / \mathrm{I}_{1}$ ratio also indicates a hydrophobic environment for the pyrene monomer.

The characteristic pyrene luminescence emissions are well known. The monomer fluorescence peaks about $398 \mathrm{~nm}$, and a broad excimer fluorescence band peaks about $470 \mathrm{~nm} .^{25,26,31}$ Monomer delayed fluorescence and excimer delayed fluores- cence peak at the same wavelengths with longer lifetimes. ${ }^{32}$ The monomer phosphorescence peaks between 550 and $600 \mathrm{~nm} .^{28,33}$

The dynamics of the photophysical processes of pyrene adsorbed onto surfaces are shown in Scheme 2, where the solid lines represent the radiative transitions and the dashed lines represent the radiationless transitions. An equilibrium exists between the monomeric pyrene (M) and the dimeric pyrene (D) in the ground state. This dimeric form of pyrene represents both the isolated ground state dimer, and the crystalline form of pyrene. The pyrene crystal has a dimeric structure with the molecules grouped in parallel sandwich-like pairs perpendicular to each other, which Birks defines as a type-B crystal. ${ }^{34}$

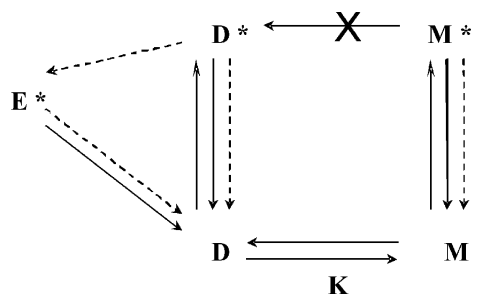

Scheme 2 Dynamics of photophysical processes of pyrene adsorbed onto calixarenes where the solid lines represent the radiative transitions and the dashed lines represent the radiationless transitions. (M: monomer; E: excimer; D: dimer; *: excited species).

Upon excitation, the excited state of the dimer ( $\left.D^{*}\right)$ is rather unstable, ${ }^{25}$ and the radiationless transition to the excimer state $\left(\mathrm{E}^{*}\right)$ can take place. For silica gel surfaces, it is assumed that the excited dimer reorients its configuration and forms an excimer during its fluorescence lifetime ${ }^{35 a}$ being the relaxation process from the excited dimer to excimer much faster that the radiative process of dimer emission. ${ }^{25,35 b}$ In other words, the fluorescence quantum yield of the excited dimer is very small compared with those of the monomer and excimer. ${ }^{35 b}$ This is the reason why the usual prompt emissions from pyrene are only the monomer and excimer emissions and also why the pyrene dimers and crystal have been assumed to present only excimer-like emissions, ${ }^{25,26,31,35}$ i.e., the dimer and crystal emissions, although present, often stay undetected.

Nevertheless, some dimer emissions can be detected and examples of dimer emissions have been reported. In cases where the motion of the pyrene molecules is restricted, as in the case

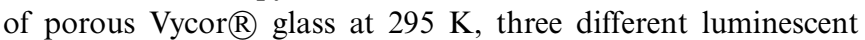
species with lifetimes of 305,105 and 20 ns were detected. ${ }^{25,36}$ These components have been assigned to the monomeric, dimeric and excimer-like species, respectively, in the order of their decreasing lifetimes. ${ }^{25,36}$

In Fig. 2, the excimer luminescence is present for the three substrates with a broad band peaking at $470 \mathrm{~nm}$. However, in the case of calix[4]arene, the maximum at $450 \mathrm{~nm}$ is predominant. We believe this $450 \mathrm{~nm}$ emission is from the pyrene dimers which exist both in the pyrene microcrystals and also (although in less extent) inside the calix[6]arene and calix[8]arene cavities.

For these reasons, along with other evidence discussed further in this paper, we added to the well known diagram in Scheme 2 (the dynamics of the photophysical processes of pyrene adsorbed onto surfaces) $)^{25,35 b}$ a solid line, representing the radiative decay from the excited dimer. 


\section{Time resolved luminescence and lifetime distribution analysis (LDA)}

Time resolved fluorescence spectra of pyrene crystals and of pyrene in the three calixarenes, excited at $337 \mathrm{~nm}$ are presented in Fig. 3. The three pyrene-calixarene samples exhibit a strong monomer emission around $400 \mathrm{~nm}$, as for pyrene crystals, the absence of the monomer fluorescence peak is notorious. This has been explained by the energy transfer from the pyrene monomer to the selftrapped dimer. ${ }^{34}$

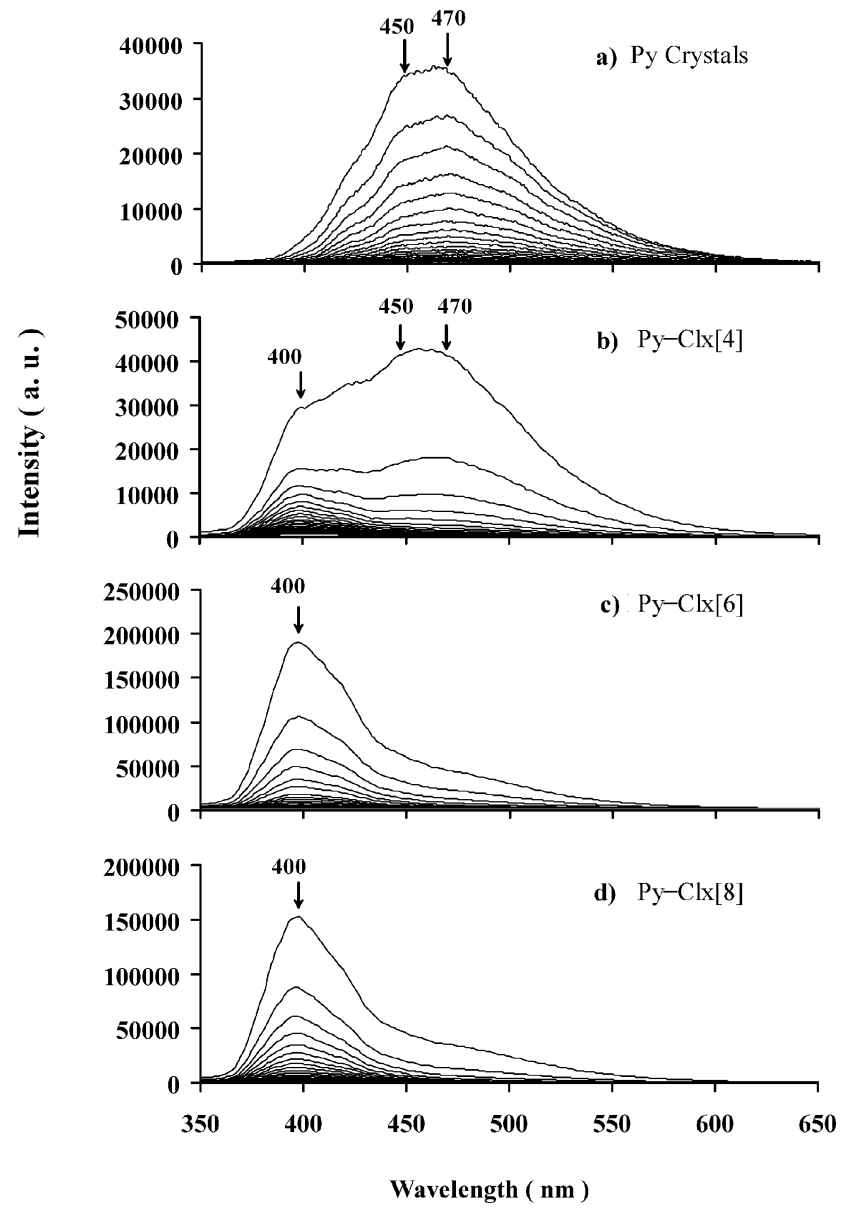

Fig. 3 Laser induced time resolved fluorescence spectra of pyrene crystals (a) and for pyrene on $p$-tert-butylcalix $[n]$ arene, $n=4,6,8$ (b, c, and d), molar ratio guest : host $1: 50$, excited at $337 \mathrm{~nm}$.

However, apart the well known broad band of the excimer peaking at $470 \mathrm{~nm}$, a $450 \mathrm{~nm}$ maximum is also present. In order to fully understand the origin of these emissions, a lifetime distribution analysis was performed. In this analysis we studied the prompt emissions of pyrene using the same method previously used by our group to study the triplet emissions of pyrene in calix[4]arene. ${ }^{28}$ The mathematical details of this approach are given in ref. 28

Lifetime distribution analysis is needed for studying pyrene included within calixarene's nanocavities since these molecules present different conformations and even different possible adsorption sites. Therefore the adsorbent is heterogeneous and continuous distributions of lifetimes provide a better description than the assumption of simple discrete lifetimes.
Model studies with this method proved that the recovery of monoexponential decays was possible even when severe noise was added to the decay. The option of changing the step of the time scale (as explained in ref. 28) enables us to eliminate computational artifacts related to the distribution widths. Therefore we can conclude that the distributions obtained for the emissions of monomers, excimers, microcrystals and dimers are genuine lifetime distributions and not monoexponentials which computational artifacts transform into pseudo-distributions.

For comparison purposes, Fig. 4 shows the lifetime distribution analysis of the time resolved decay data for pyrene crystals (a) and for pyrene within the three hosts under study (b, c and d).

Table 1 presents the mean lifetime and the full width half maximum (fwhm) obtained for each distribution in pyrene crystals, and in pyrene-calixarene [4], [6] and [8] samples. The mean lifetime is presented in nanoseconds, the fwhm is presented in $\log (\tau)$, due to the logarithmic time scale, and relative error estimates are provided in percentages, to facilitate reading. The obtained distributions for the pyrene crystals at a $450 \mathrm{~nm}$ observation wavelength confirmed the absence of pure monomer emissions (usually with 200-400 ns lifetimes). The two emitting species are the crystal and excimer with lifetimes of 6 and $20 \mathrm{~ns}$, respectively. The much weaker emission of the crystal agrees with the arguments for the crystal's low luminescence quantum yield cited above..$^{25,35 b}$

For calix[6]arene and calix[8]arene (Fig. 4 (c) and (d), respectively), three different lifetime distributions were obtained. The recovered lifetimes are comparable to those obtained for Vycor $\AA$ glass $^{36}$ (mentioned earlier) and can be assigned in the same way to the monomeric, dimeric and excimer-like species, respectively, in the order of their decreasing lifetimes. In the case of calix[4]arene (Fig. 4(b), the three lifetimes obtained correspond to the crystal, excimer, and monomer emissions with 5,14 and 183 ns lifetimes, respectively.

Table 1 Mean lifetime (in ns) and full width half maximum (in $\log (\tau)$ ), with the respective relative error estimates (in percentages), obtained for each distribution in pyrene crystals, and in pyrene-calixarene [4], [6] and [8] samples

\begin{tabular}{|c|c|c|c|c|}
\hline & Crystals & Monomer & Excimer & Dimer \\
\hline \multicolumn{5}{|l|}{ Py Crystals } \\
\hline Mean lifetime/ns & 6 & & 20 & \\
\hline Error estimate (\%) & 5.5 & & 2.4 & \\
\hline Fwhm & 2.9 & & 0.24 & \\
\hline Error estimate (\%) & 0.34 & & 29 & \\
\hline \multicolumn{5}{|l|}{ Py-Clx $[4]$} \\
\hline Mean lifetime/ns & 5 & 183 & 14 & \\
\hline Error estimate (\%) & 1.1 & 0.71 & 5.9 & \\
\hline Fwhm & 0.31 & 0.13 & 2.5 & \\
\hline Error estimate (\%) & 13 & 23 & 0.40 & \\
\hline \multicolumn{5}{|l|}{ Py-Clx $[6]$} \\
\hline Mean lifetime/ns & & 188 & 18 & 65 \\
\hline Error estimate (\%) & & 3.5 & 2.6 & 5.3 \\
\hline Fwhm & & 0.32 & 1.6 & 0.57 \\
\hline Error estimate (\%) & & 28 & 13 & 28 \\
\hline \multicolumn{5}{|l|}{ Py-Clx $[8]$} \\
\hline Mean lifetime/ns & & 267 & 20 & 130 \\
\hline Error estimate (\%) & & 1.2 & 4.5 & 6.2 \\
\hline Fwhm & & 0.21 & 0.64 & 0.41 \\
\hline Error estimate (\%) & & 19 & 22 & 22 \\
\hline
\end{tabular}



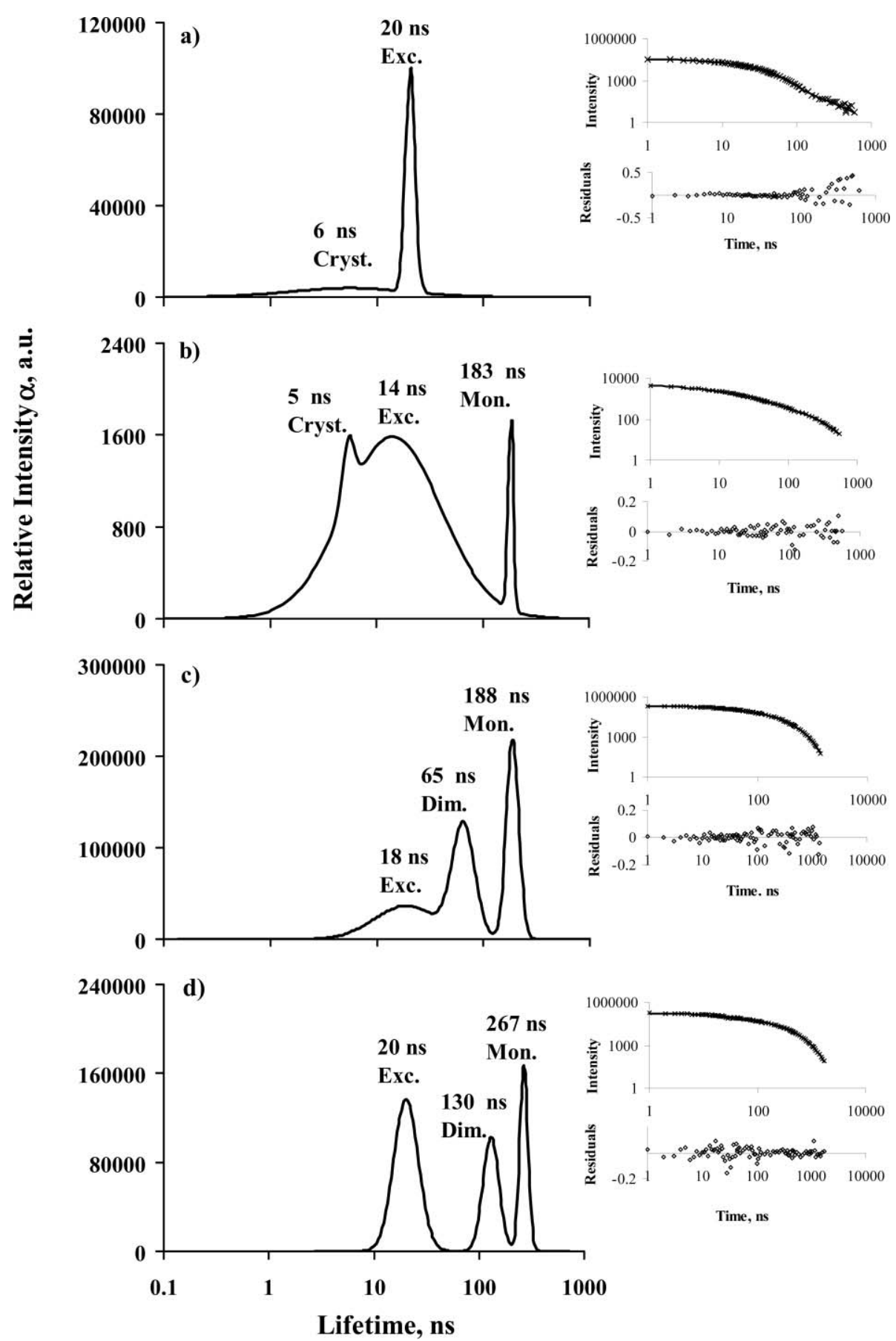

Fig. 4 Lifetime distributions for pyrene crystals (a) and for pyrene on $p$-tert-butylcalix $[n]$ arene, $n=4,6$ and 8 (b, c, and d, respectively) recovered from time resolved luminescence decays observed at 450 (a) and $404 \mathrm{~nm}$ (b, c and d). The insets show the fitting of the recovered decay to the experimental data and the relative residuals.

The lifetimes of the same emissive species vary with the different characteristics of the three hosts although they are in the same magnitude. The monomer lifetime for calix[4]arene is about 183 ns whereas for calix[6]arene and calix[8]arene it is about 188 and $267 \mathrm{~ns}$, respectively. This increase in monomer lifetime for calix[8]arene is probably due to some conformations that can be assumed by this host, in which the pyrene monomer experiences tighter conditions inside the cavity. ${ }^{9-12}$ The excimer lifetimes of 14, 18 and $20 \mathrm{~ns}$ for calix[4]arene, calix[6]arene and calix[8]arene, respectively, are less affected by the size of the cavity because the excimer formation process already implies a certain mobility of the pyrene molecules to achieve a specific excimer conformation. The emission of the dimer is detected for calix[6]arene and calix[8]arene with lifetimes of 65 and $130 \mathrm{~ns}$, respectively, also reflecting the differences of the hosts cavities.

Hence, the $450 \mathrm{~nm}$ emission detected in the SSL spectra results, in the case of calix[4]arene, from pyrene microcrystals and in 
the case of calix[6]arene and calix[8]arene, from pyrene dimers constrained inside the cavity.

This interpretation is consistent with GSDR results (see above) that suggest the presence of ground state dimers (BGSAs) trapped inside the cavities of calix[6]arene and calix[8]arene, but not of calix[4]arene. These results also agree with previous studies of pyrene adsorbed onto calix[4]arene, ${ }^{28}$ where the presence of pyrene microcrystals was confirmed, but no evidence for the presence of other ground state dimers was found. At the observation wavelength of $470 \mathrm{~nm}$ the lifetime distribution analysis (data not shown) also confirmed the absence of the dimer for the calix[4]arene.

In all cases where a dimer is not very tightly included into the calixarene's cavity, it may also achieve the excimer conformation and emit its excimer fluorescence, although being affected by the confinement effect of the cavity. Therefore the final conformation and orientation of the excimer may vary slightly, causing the excimer decay to assume a distribution of lifetimes, similarly to the case of the emissions of monomers and dimers.

The monomer phosphorescence previously reported for calix[4]arene with air equilibrated samples ${ }^{28}$ was only visible for calix[6]arene with argon purged samples. In the case of calix[8]arene, this emission, although visible in air equilibrated samples, became slightly stronger with the argon purging. The lifetime distribution analysis at the observation wavelength of the monomer phosphorescence, $600 \mathrm{~nm}$, revealed, as for calix[4]arene, ${ }^{28}$ the presence of both the delayed excimer fluorescence and monomer phosphorescence. The lifetimes obtained for the delayed excimer fluorescence and for the monomer phosphorescence were $71 \mu \mathrm{s}$ and $2.5 \mathrm{~ms}$, respectively for calix[6]arene and $240 \mu \mathrm{s}$ and $11 \mathrm{~ms}$, respectively for calix[8]arene (data not shown).

\section{Diffuse reflectance laser flash photolysis (DRLFP)}

Fig. 5(a) and (b) present the air equilibrated transient absorption spectra of pyrene adsorbed onto calix[4]arenes and calix[6]arene, respectively, in the $1: 10$ pyrene : calixarene molar ratios. For the py-calix[4]arene sample, the two strong absorptions around 320 and $400 \mathrm{~nm}$, are characteristic of the host. In fact, the spectra of the three pure hosts are identical ${ }^{19 a}$ and present the same shape and relative intensities shown in the inset of Fig. 5(a). The direct excitation at $355 \mathrm{~nm}$ of the pure calixarene results in the formation of the phenoxyl radical of the calixarene with its characteristic absorption band around $400 \mathrm{~nm}^{19 a}$ :

$$
\mathrm{CLX}[n] \mathrm{OH} \stackrel{h v,-\mathrm{H}^{\bullet}}{\longrightarrow} \mathrm{CLX}[n] \mathrm{O}^{*}, n=4,6 \text { and } 8 .
$$

(In this equation, one of the hydroxyl groups of the p-tertbutylcalix $[n]$ arenes was set out $-\mathrm{CLX}[n] \mathrm{OH}-$ in order to show the formation of the phenoxyl radical from the calixarene.)

The pyrene radical cation absorption band at $450 \mathrm{~nm}^{37}$ and the bands of the triplet state of pyrene at 415,480 and $515 \mathrm{~nm}^{37}$ are present, and they become more evident for higher concentrations of pyrene. In low concentrated samples, these bands are masked by the strong absorption of the phenoxyl radical around $400 \mathrm{~nm}$. The presence of the triplet state of pyrene in this host is consistent with the previous report of monomer phosphorescence for calix[4]arene ${ }^{28}$ even in air equilibrated samples.
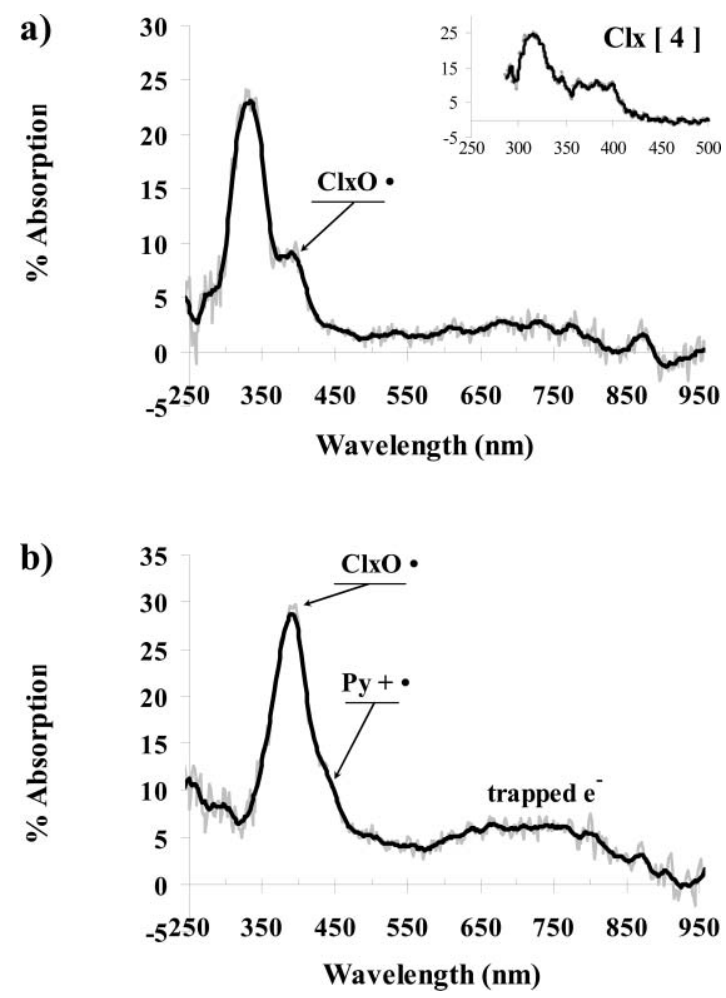

Fig. 5 Smoothed (black) and obtained (grey) transient absorption spectra of Py on calix[4]arene and calix[6]arene, (a) and (b), respectively, in the molar ratio of $1: 10$ (mol : mol). The spectra were collected $20 \mu$ s after the laser pulse with the excitation wavelength at $355 \mathrm{~nm}$. The inset shows the transient absorption spectra of pure calix[4]arene excited at $355 \mathrm{~nm}$.

In the case of calix[6]arene, the absorption around $320 \mathrm{~nm}$ decreases while the $\sim 400 \mathrm{~nm}$ band increases dramatically. The outstanding growth of this phenoxyl radical band ${ }^{19 a, d, e}$ suggests a very favourable situation for the hydrogen atom abstraction to occur, as will be discussed later. A small cation radical band is also present at $450 \mathrm{~nm},{ }^{37}$ although masked by the stronger absorption at $400 \mathrm{~nm}$. The broad band in the $500-800 \mathrm{~nm}$ region is related to presence of trapped electrons, ${ }^{37 a, 38 a, b}$ resulting from the formation of the pyrene radical cation. In some cases the phenoxyl radical spectrum alone also presents a broad and low intensity band in this region. ${ }^{38 c}$ This is not the case in this system. The absorption spectrum of the pure calixarene (see inset of Fig. 5(a)) presents the phenoxyl absorption at $400 \mathrm{~nm}$, but not the broad band (from $500-950 \mathrm{~nm}$ zero \% absorption was detected). Besides this, the decay kinetics of the broad band is different from that of the phenoxyl radical transient absorption. Therefore, in this case, this broad band is not due to the presence of the phenoxyl radical.

Radical formation and trapped electron transient absorption spectra do not change within experimental error when the excitation energy of the laser varies from 10 to $30 \mathrm{~mJ}$ per pulse. By contrast, trapped electron transient absorption spectra were verified to be highly dependent on the sample's humidity content.

The triplet absorption bands at 415, 480 and $515 \mathrm{~nm}^{37}$ become more evident with the argon purged sample (data not shown). This is consistent with the presence of a pyrene dimer forcing the calix[6]arene's cavity to be wider and less protective than it would be when accommodating only one pyrene molecule. It is also consistent with time resolved luminescence results which 
have shown a much stronger monomer phosphorescence with air equilibrated samples for calix[4]arene than for calix[6]arene or even calix[8]arene.

For calix[8]arene (data not shown) the results are similar to calix[6]arene, but the growth of the phenoxyl absorption band is not so evident which means that hydrogen atom abstraction occurs but does not occur as much as for calix[6]arene. The argon purging doesn't increase the triplet yield significantly and this is again due to the multiplicity of conformations in which pyrene molecules can be accommodated inside the calix[8]arene's cavity. However, the same weak radical cation and trapped electron absorptions can be detected at $450 \mathrm{~nm}$, and from 500 to $800 \mathrm{~nm}$, respectively. ${ }^{37 a, 38 a, b}$

Diffuse reflectance ground state absorption spectra clearly show that both for 266 or $355 \mathrm{~nm}$ laser or lamp excitation, pyrene is the compound which is preferentially excited. No difference in experimental transient absorption spectra was detected and at the same time, there is no experimental evidence for different photodegradation with the excitation wavelength. Also, energy dependence experiments were performed and again transient absorption spectra were recorded. The final conclusion was that transient absorption spectra were the same within experimental error in the 10 to $30 \mathrm{~mJ}$ per pulse excitation range; therefore the final photodegradation products should also be similar.

\section{Irradiation and photodegradation products}

The analyses of the degradation products by HPLC and GCMS, shown in Fig. 6, revealed that hydroxypyrene is a major photodegradation product for the three hosts studied. The assignment was confirmed by comparison with an authentic sample. The results also indicated that the concentration of hydroxypyrene was much higher in the case of calix[6]arene.

The mechanism for the formation of hydroxypyrene was first proposed by Mao and Thomas ${ }^{39 a}$ for alumina and silica-alumina substrates, and later reported by Dabestani et al. ${ }^{39 b}$ for silica surfaces and by Oliveira et al. ${ }^{29}$ for cellulose. This mechanism is also consistent with our results, obtained for calixarenes as hosts.

In accordance with this mechanism (see Scheme 3), the pyrene radical cation is formed and reacts with water molecules (which are present in residual amounts in most samples), to form hydroxypyrene. Calix[6]arene was the host where the concentration of hydroxypyrene was found to be the highest, followed by calix[8]arene and calix[4]arene, in the order of the decreasing concentrations of this photodegradation product. In the same way calix[6]arene is also the host where the transient absorptions of pyrene radical cation and of the resulting trapped electron were stronger. The intensity of these absorption bands also decreases in the same order for calix[8]arene and calix[4]arene.<smiles>c1cc2ccc3ccc-2c2c(c1)cccc32</smiles>

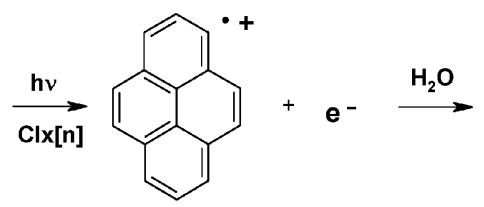<smiles>Oc1ccc2ccc3cccc4ccc1c2c34</smiles>

Scheme 3 Formation of hydroxypyrene in calixarenes.

The retention times of hydroxypyrenes are considerably shorter than that of pyrene in the HPLC column. ${ }^{39}$ The used HPLC column has a non-polar stationary phase (RP-18). Therefore more polar compounds are expected to show lower retention times when using a polar mobile phase, which is the reason why the retention time of hydroxypyrene is lower than that of pyrene (see Fig. 6). The retention time of the fraction $\mathrm{F}$ is even lower than that of hydroxypyrene, indicating that this compound is more polar than hydroxypyrene. The inset in Fig. 6 shows the absorption

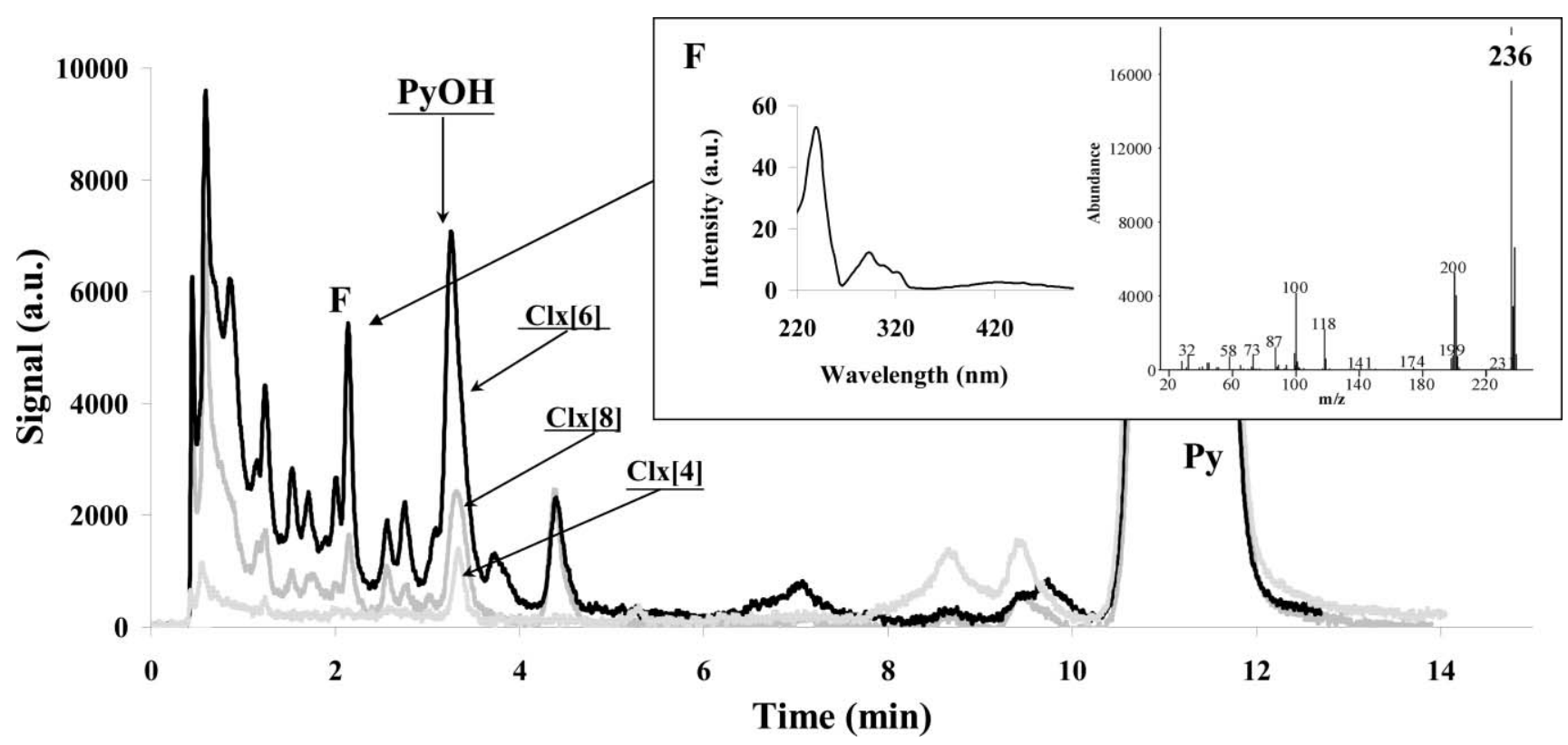

Fig. 6 HPLC chromatogram of an irradiated sample of Py in calix[4]arene, calix[6]arene and calix[8]arene. The inset shows the UV/Vis spectrum and a mass spectrum obtained by GC-MS of one of the degradation products. 
and GC-MS spectra of this fraction. The analysis of the mass spectrum indicates that the molecular ion shows a $m / z=236$. This compound is not expected to contain carbonyl groups since the loss of $m / z=28$ (loss of CO) was not detected in the fragmentation pattern.

In their work, Mao and Thomas used HPLC and GC-MS in the same way and detected both the first fraction of hydroxypyrene, with a mass number of $218(\mathrm{~m} / \mathrm{z})$, and this last fraction with a mass number of $236(\mathrm{~m} / \mathrm{z}) .{ }^{39 a}$ They suggested a biphotonic mechanism for the formation of dihydroxypyrene $\left[\mathrm{Py}(\mathrm{OH})_{2}\right]$, and studied the $\mathrm{pH}$ absorption dependence of this second fraction. Dabestani et al. also identified the presence of hydroxypyrene for pyrene in silica gel and reported the formation of pyrenediones [mass number $232(\mathrm{~m} / \mathrm{z})$ ], resulting from the oxidation of dihydroxypyrene. ${ }^{39 b}$ However, the compound in our fraction $\mathrm{F}$ cannot be $\mathrm{Py}(\mathrm{OH})_{2}$ because the mass number of this compound is $236(\mathrm{~m} / \mathrm{z})$ whereas the mass number of dihydroxypyrene is $234(\mathrm{~m} / \mathrm{z})$.

Transient absorption results have shown that the formation of the phenoxyl radical of the calixarene by the abstraction of a hydrogen atom is also very strong for calix[6]arene, less strong for calix[8]arene and not present for calix[4]arene. In the same way, this fraction $\mathrm{F}$ is only present in the samples of calix[6]arene and calix[8]arene, and not for calix[4]arene (see Fig. 6). Within calix[6]arene and calix[8]arene, pyrene molecules seem to be particularly close to the hydroxyl groups of the lower rims of the calixarene and to favour the hydrogen abstraction resulting in the phenoxyl radical formation. The reaction of a diphenylanthracene radical cation with water in acetonitrile has been reported to result in the formation of dihydro-dihydroxy-diphenylanthracene. ${ }^{40} \mathrm{~A}$ similar reaction mechanism could be expected for $\mathrm{Py}^{+}$in these surfaces. Hence, we tentatively assign this compound as dihydrodihydroxypyrene.

\section{Conclusions}

Pyrene molecules form inclusion complexes with the three calixarene hosts under study. For calix[4]arene, only a monomer fits inside the cavity, and for the most concentrated samples, the presence of microcrystals outside the cavity (with a weak prompt emission) was detected.

The cavities of calix[6]arene and calix[8]arene accommodate not only the pyrene monomer but also a ground state dimer. This feature is much more evident in the case of calix[6]arene. The multiplicity of possible conformations for calix[8]arene decreases the number of host molecules available to accommodate the dimer.

These ground state dimers, in the cavity's restricted environment become luminescent.

The presence of a pyrene radical cation and trapped electrons was detected for the three hosts. The increase in the formation of the phenoxyl radical of the calixarene resulting from the direct excitation of the calixarene is particularly strong for the calix[6]arene, and is also present for calix[8]arene.

Hydroxypyrene was detected as the main photodegradation product of pyrene for the three hosts under study. Dihydrodihydroxypyrene was also formed in the case of calix[6]arene and calix[8]arene. The formation of the calixarene's phenoxyl radical and subsequent hydrogen abstraction is consistent with the formation of this photodegradation product.

\section{Acknowledgements}

Tiago Branco thanks F. C. T. for a doctoral fellowship SFRH/BD/8143/2002.

\section{References}

1 C. D. Gutshe, Calixarenes, Royal Society of Chemistry, Cambridge, UK, 1989; C. D. Gutshe, Calixarenes Revisited, Monographs in Supramolecular Chemistry, Springer Verlag, Berlin, 1998.

2 Calixarenes in Action, ed. L. Mandolini and R. Ungaro, Imperial College Press, London, 2000.

3 Calixarenes, ed. J. Vincens and V. Böhmer, Kluwer Academic Press, Dordrecht, 1991

4 A. Ikeda and S. Shinkai, Novel cavity design using calix[n]arene skeletons: toward molecular recognition and metal binding, Chem. Rev., 1997, 97, 1713-1734.

5 R. Perrin, R. Lamartine and M. Perrin, The potential industrial applications of calixarenes, Pure Appl. Chem., 1993, 65, 1549-1559.

6 I. Alam and C. D. Gutsche, Calixarenes. 24. Complexation by water soluble calixarenes, J. Org. Chem., 1990, 55, 4487-4489.

7 M. Lazzarotto, F. Nachtigall and F. Nome, Calixarenos: Receptores para Reconhecimento Molecular, Quim. Nova, 1995, 18, 444-451, in Portuguese;; T. Cairns and G. Eglinton, Hydrogen bonding in phenols, Nature, 1962, 196, 535-537.

8 C. D. Gutsche and I. Alam, Calixarenes. 23. The complexation and catalytic properties of water soluble calixarenes, Tetrahedron, 1988, 44, 4689-4694.

9 C. Gutsche and L. Bauer, Calixarenes. 13. The conformational properties of calix[4]arenes, calix[6]arenes, calix[8]arenes, and oxacalixarenes, J. Am. Chem. Soc., 1985, 107, 6052-6059.

$10 \mathrm{~T}$. Liang and $\mathrm{K}$. Laali, On the utility of ${ }^{13} \mathrm{C}$ (cpmas) NMR in conformational studies of simple hydroxy-, alkoxy- and acetoxycalixarenes in the solid state, Chem. Ber., 1991, 124, 2637-2639.

11 K. Araki, K. Akao, H. Otsuka, K. Nakashima, F. Inokuchi and S. Shinkai, Immobilization of the ring inversion motion in calix[6]arene by a cap with $\mathrm{c}_{3}$-symmetry, Chem. Lett., 1994, 1251-1254.

12 G. Andreetti, R. Ungaro and A. Pochini, Crystal and molecular structure of cyclo\{quater[(5-t-butyl-2-hydroxy-1,3-phenylene)methylene]\} toluene (1: 1) clathrate, Chem. Commun., 1979, 1005-1007; M. Halit, D. Oehler, M. Perrin, A. Thozet, R. Perrin, J. Vicens and M. Bourakhouadar, J. Inclusion Phenom. Macrocyclic Chem., 1988, 6, 613-623; C. Gutsche, A. Gutsche and A. Karaulov, Calixarenes 11. Crystal and molecular structure of $p$-tert-butylcalix[8]arene, J. Inclusion Phenom. Macrocyclic Chem., 1985, 3, 447-451.

$13 \mathrm{~K}$. Udachin, G. Enright, E. Brouwer and J. Ripmeester, tButylcalix[4]arene compounds with long chain guests: structures and host-guest interactions, J. Supramol. Chem., 2001, 1, 97-100.

14 S. Shinkai, K. Araki and O. Manabe, Does the calixarene cavity recognise the size of guest molecules? On the hole-size selectivity in water-soluble calixarenes, J. Chem. Soc., Chem. Commun., 1988, 3, 187189; S. Shinkai, Calixarenes - the third generation of supramolecules, Tetrahedron, 1993, 49, 8933-8968.

15 A. F. D. Namor, R. G. Hutcherson, F. J. S. Velarde, M. L. ZapataOrmachea, L. E. P. Salazar, I. Jammaz and N. Rawi, An overview on the solution thermodynamics of lower rim functionalised calixarene derivatives and metal cations. New derivatives containing amino and thioalkyl functional groups, Pure Appl. Chem., 1998, 70, 769-778.

16 D. Diamond and K. Nolan, Calixarenes: designer ligands for chemical sensors, Anal. Chem., 2001, 1, 23a-29a.

17 D. M. Rudkevich, Nanoscale molecular containers, Bull. Chem. Soc. Jpn., 2002, 75, 393-413.

18 R. Dabestani, Photophysical and photochemical behaviour of polycyclic aromatic hydrocarbons on silica surfaces, Inter-Am. Photochem. Soc. Newsl., 1997, 20, 24-36; R. Dabestani and I. N. Ivanov, A compilation of physical, spectroscopic and photophysical propertiesof polycyclic aromatic hydrocarbons, Photochem. Photobiol., 1999, 70, $10-34$.

19 L. F. Vieira Ferreira, M. R. Vieira Ferreira, A. S. Oliveira, T. J. F. Branco, J. V. Prata and J. C. Moreira, Diffuse reflectance studies of beta-phenylpropiophenone and benzophenone inclusion complexes with calix[4], [6] and [8] arenes, Phys. Chem. Chem. Phys., 2002, 4, 204-210; L. F. Vieira Ferreira, M. R. Vieira Ferreira, J. P. Da Silva, 
I. Ferreira Machado, A. S. Oliveira and J. V. Prata, A novel laserinduced luminescence resulting from benzophenone/ $O$-propylated $p$ tert-butylcalix[4]arenes complexes. A diffuse reflectance study, Photochem. Photobiol. Sci., 2003, 2, 1002-1010.

20 L. F. Vieira Ferreira, J. C. Netto-Ferreira, I. Khmelinskii, A. R. Garcia and S. M. B. Costa, Photochemistry on surfaces: matrix isolation mechanisms for study of interactions of benzophenone adsorbed on microcrystalline cellulose investigated by diffuse reflectance and luminescence techniques, Langmuir, 1995, 11, 231-236.

21 L. F. Vieira Ferreira, M. R. Freixo, A. R. Garcia and F. Wilkinson, Photochemistry on surfaces: fluorescence quantum yield determination of dyes adsorbed on microcrystalline cellulose, J. Chem. Soc., Faraday Trans., 1992, 88, 15-22; W. W. Wendlandt and H. G. Hecht, in Reflectance Spectroscopy, ed. P. J. Elving and I. M. Kolthoff, Interscience Publishers, New York, 1969, pp. 114-118.

22 L. F. Vieira Ferreira, S. M. B. Costa and E. J. Pereira, Fluorescence quantum yield evaluation of strongly absorbing dye solutions as a function of the excitation wavelength, J. Photochem. Photobiol., A, 1991, 55, 361-376.

23 A. M. Botelho do Rego and L. F. Vieira Ferreira, in Handbook of Surfaces and Interfaces of Materials, ed. H. S. Nalwa, Academic Press, 2001, vol. 2, ch. 7, pp. 275-313; L. F. Vieira Ferreira, A. S. Oliveira and J. C. Netto-Ferreira, in Fluorescence Microscopy and Fluorescent Probes 3, ed. A. Kotyc, Espero Publishing, Prague, 1999, 199-208.

24 J. P. Da Silva, L. F. Vieira Ferreira, A. M. Da Silva and A. S. Oliveira, A comparative study of the photophysics and photochemistry of 4chlorophenol adsorbed on silicalite and beta-cyclodextrin, J. Photochem. Photobiol., A, 2002, 151, 157-164.

25 N. Negishi, T. Fujii and M. Anpo, Characteristics of the photoluminescence properties of pyrene adsorbed on and doped into silica-based materials, Heterogen. Chem. Rev., 1994, 1, 231-241.

26 K. Hara, P. De Mayo, W. R. Ware, A. C. Weedon, G. S. K. Wong and K. C. Wu, Biphasic photochemistry: time-resolved spectra of adsorbed hydrocarbons, Chem. Phys. Lett., 1980, 69, 105-108; R. Bauer, P. De Mayo, W. Ware and K. Wu, Surface photochemistry. The photophysics of pyrene adsorbed on silica gel, alumina, and calcium fluoride, J. Phys. Chem., 1982, 86, 3781-3789; J. K. Thomas, Physical aspects of photochemistry and radiation chemistry of molecules adsorbed on $\mathrm{SiO}_{2}, \gamma-\mathrm{Al}_{2} \mathrm{O}_{3}$, zeolites, and clays, Chem. Rev., 1993, 93, 301-320.

27 C. H. Lochmuller and T. J. Wenzel, Spectroscopic studies of pyrene at silica interfaces, J. Phys. Chem., 1990, 94, 4230-4235.

28 T. J. F. Branco, A. M. Botelho do Rego, I. Ferreira Machado and L. F. Vieira Ferreira, A luminescence lifetime distributions analysis in heterogeneous systems by the use of Excel's Solver, J. Phys. Chem. B, 2005, 109, 15958-15967.

29 A. S. Oliveira, L. F. Vieira Ferreira, J. P. Da Silva and J. C. Moreira, Surface photochemistry: Photodegradation study of pyrene adsorbed onto microcrystalline cellulose and silica, Int. J. Photoenergy, 2004, 6, 205-213.

30 D. S. Karpovich and G. J. Blanchard, Relating the polarity-dependent fluorescence response of pyrene to vibronic coupling. Achieving a fundamental understanding of the py polarity scale, J. Phys. Chem., 1995, 99, 3951-3958; M. Chaudhuri and S. Ganguly, Polarized absorption and fluorescence spectra of crystalline pyrene, J. Phys. C: Solid State Phys., 1970, 3, 1791-1796.

$31 \mathrm{~K}$. Matsui and N. Usuki, Excimer fluorescence of pyrene in sol-gel silica, Bull. Chem. Soc. Jpn., 1990, 63, 3516-3520.

$32 \mathrm{~L}$. Li, Z. Zhang, W. Long and A. Tong, Study of properties on nonprotected room temperature phosphorescence and delayed excimer fluorescence of pyrene solution, Spectrochim. Acta, Part A, 2001, 57, 385-393; B. Srinivasan, M. Kinoshita and S. McGlynn, Delayed luminescence of organic mixed crystals. VI. Delayed excimer fluorescence of pyrene in biphenyl, J. Chem. Phys., 1967, 47, 5090-5096.
33 O. Gijzeman, J. Langelaar and J. Van Voorst, Triplet excimer emission from pyrene single crystals, Chem. Phys. Lett., 1970, 5, 269-272; L. Peter and G. Vaubel, Phosphorescence of pure crystalline pyrene, Chem. Phys. Lett., 1973, 21, 158-160; G. Romanovskaya, M. Koroleva, V. Nikashina, B. Zuev and B. Myasoedov, Polyacrylic fiber filled with clinoptilolite for determination of polycyclic aromatic hydrocarbons by room-temperature phosphometry, Dokl. Phys. Chem., 2002, 383, 78-80; A. Sal'nikov, A. Spivak and G. Mel'nikov, Phosphorescence of pyrene molecules in the agar solid matrix at room temperature, Russ. Phys. J., 2002, 45, 570-573; A. Castillo, A. Carretero, J. Fernández, W. Jin and A. Gutiérrez, Heavy atom induced room temperature phosphorescence: a tool for the analytical characterization of polycyclic aromatic hydrocarbons, Anal. Chim. Acta, 2004, 516, 213-220.

34 B. Stevens, Some effects of molecular orientation on fluorescence emission and energy transfer in crystalline aromatic hydrocarbons, Spectrochim. Acta, 1962, 18, 439-448; J. B. Birks, Photophysics of Aromatic Molecules, Wiley, London, 1970.

35 T. Fugii and E. Shimizu, Photophysics on solid surfaces. Ground state configuration of dimeric pyrene in the adsorbed state on a silica gel surface and geometrical relaxation for excimer formation, Chem. Phys. Lett., 1987, 137, 448-452; T. Fujii, E. Shimizu and S. Suzuki, Photophysics at solid surfaces. Evidence of dimer formation and polarization of monomer and excimer fluorescences of pyrene in the adsorbed state on silica-gel surfaces, J. Chem. Soc., Faraday Trans. 1, 1988, 84, 4387-4395; Y. Tozuka, E. Yonemoshi, T. Ogushi and $\mathrm{K}$. Yamamoto, Fluorometric studies of pyrene adsorption on porous crystalline cellulose, J. Colloid Interface Sci., 1998, 205, 510-515.

36 T. Fujii, A. Ishii, H. Satozono, S. Suzuki, M. Che and M. Anpo, Photophysics on solid surfaces. Time-resolved fluorescence spectra of pyrene adsorbed on calcinated vycor glass, Bull. Chem. Soc. Jpn., 1990, 63, 2475-2480.

37 K. K. Iu and J. K. Thomas, Single-photon ionization of pyrene and anthracene giving trapped electrons in alkali-metal cation-exchanged zeolites X and Y: a direct time-resolved diffuse reflectance study, J. Phys. Chem., 1991, 95, 506-509; S. Pankasem and J. K. Thomas, Reflectance spectroscopic studies of the cation radical and the triplet of pyrene on alumina, J. Phys. Chem., 1991, 95, 6990-6996; X. Liu, K. Iu and J. K. Thomas, Photophysical properties of pyrene in zeolites. Observation of pyrene anion radicals in zeolites x and y, Chem. Phys. Lett., 1993, 204, 163-167; G. Zanini, H. Montejano, J. Carlos and C. Previtali, Solvent effects on intermolecular photoinduced electron transfer reactions of aromatic molecules, J. Photochem. Photobiol., A, 1997, 109, 9-14; G. Zhang and J. K. Thomas, Photoinduced charge-transfer reaction between pyrene and $n, n^{\prime}$-dimethylaniline on silica gel surfaces, J. Phys. Chem. B, 1997, 101, 8569-8577.

38 K. Lednev, N. Mathivanan and L. J. Johnston, Photochemistry of stilbene adsorbed on silica gel and $\mathrm{NaX}$ zeolite. A diffuse reflectance laser flash photolysis study, J. Phys. Chem., 1994, 98, 11444-114451; E. H. Ellison, Electron trapping in polar-solvated zeolites, J. Phys. Chem. B, 2005, 109, 20424-20432; L. J. Johnston, N. Mathivanan, F. Negri and W. Siebrand, Assignment and vibrational analysis of the 600 nmabsorption band in the phenoxyl radical and some of its derivatives, Can. J. Chem., 1993, 71, 1655-1662.

39 Y. Mao and J. K. Thomas, Photochemical reactions of pyrene on surfaces of $\gamma$-alumina and silica-alumina, Langmuir, 1992, 8, 25012508; C. Reyes, M. Medina, C. Crespo-Hernandez, M. Cedeno, R. Arce, O. Rosario, D. Steffenson, I. Ivanov, M. Sigman and Reza Dabestani, Photochemistry of pyrene on unactivated and activated silica surfaces, Environ. Sci. Technol., 2000, 84, 415-421.

40 R. E. Sioda, Electrolytic Oxidation of 9,1 O-Diphenylanthracene and Properties of Its Free Radical Cation and Anion, J. Phys. Chem., 1968, 72, 2322-2330. 